Bruno Mario Cesana

\section{Negative randomized clinical trials (RCTs): further insight from the biostatistician's point of view}

Accepted: 14 October 2015

Published online: 21 October 2015

(C) Springer-Verlag Berlin Heidelberg and ESICM 2015

Dear Editor,

Gattinoni and Giomarelli's [1] con-

clusion is that "negative" randomized

controlled trials (RCTs) occur

"...when they are too generic or wrong". However, I think that some insights have to be added from the biostatistician's point of view.

It is well known that RCTs are based on a null hypothesis $\left(\mathrm{H}_{0}\right)$ of no difference and on an alternative hypothesis $\left(\mathrm{H}_{\mathrm{A}}\right)$ quantified on the "minimal clinical relevant difference $(\delta)$ " (superiority RCTs) or on the "maximal difference not clinically relevant" (non-inferiority and equivalence RCTs) or, better, for continuous variables, on the effect size: $\delta$ divided by the variability of the investigated phenomenon $(\sigma)$.

Then, after having fixed the significance level ( $\alpha=0.05$, two tailed, usually) and the pertinent statistical test, it is possible to calculate the sample size (to be reported in the paper [2]) for having a satisfactory probability (power, 0.80 , at least) of rejecting $\mathrm{H}_{0}$. Of course, if the $\mathrm{H}_{\mathrm{A}}$ is true with a larger difference (effect size) than the foreseen one, the probability of rejecting $\mathrm{H}_{0}$ will be greater and vice versa if the true difference (effect size) is lower.

Then, according to the frequentist approach [3], it is expected that, if the foreseen $\mathrm{H}_{\mathrm{A}}$ is "true", it will be demonstrated in about the $80 \%$ of the cases (power, correct rejection of a "false" $\mathrm{H}_{0}$ ) and in the remaining about $20 \% \mathrm{H}_{0}$ will not be disproved ( $\beta$, type II error). Finally, the probability of wrongly rejecting a "true" $\mathrm{H}_{0}$ ( $\alpha$, type I error) is equal to the fixed significance level $(\alpha)$.

A very frequent cause of "negative" RCTs is an overoptimistic $\mathrm{H}_{\mathrm{A}}$, often for having a lower number of patients to enrol into the RCTs; indeed, two "negative" trials (see references 8 and 10 in [1]) supposed a too high $20 \%$ decrease of the mortality in favour of the prone position vs. the supine position.

Finally, it has to be said that researchers tend to wrongly interpret the significance value as the probability that the $\mathrm{H}_{0}$ is "true" instead of, correctly, the probability of having obtained the result only by chance. In fact, in order to calculate the probability that $\mathrm{H}_{0}$ is "true", one has to consider that the $\mathrm{H}_{0}$ (and also the $\mathrm{H}_{\mathrm{A}}$ ) has "a priori" probability of being "true" ranging, in theory, from 0.00 to 1.00 . So, by multiplying the "a priori" probability of a "true" $\mathrm{H}_{0}$ by the "Bayes factor" that one can calculate from the significance $p$ value $[4,5]$, it is possible to obtain the "a posteriori" probability of $\mathrm{H}_{0}$ being true. For example, statistically significant $p$ values of 0.05 decrease the "a priori" probability of 0.05 (very unfavourable), 0.50 (equipoise-like or toss-up) and 0.95 (very favourable) to about $0.013,0.205$ and 0.831 , respectively. So, the probability of a significant result can be sensibly used to critically consider the probability that $\mathrm{H}_{0}$ is actually "true".

Compliance with ethical standards

Conflicts of interest None.

\section{References}

1. Gattinoni L, Giomarelli P (2015) Acquiring knowledge in intensive care: merits and pitfalls of randomized controlled trials. Intensive Care Med 41:1460-1464. doi: 10.1007/s00134-015-3837-7

2. Schulz KF, Altman DG, Moher D, CONSORT Group (2010) CONSORT 2010 Statement: updated guidelines for reporting parallel group randomized trials. Ann Inter Med 152:1-7

3. Neyman J, Pearson ES (1933) On the problem of the most efficient tests of statistical hypotheses. Philos Trans Roy Soc A 231:289-337

4. Goodman SN (2001) Of P-values and Bayes: a modest proposal. Epidemiology 12:295-297

5. Goodman SN (1999) Ann Intern Med 130:1005-1013

B. M. Cesana (®)

DMMT, Università degli Studi di Brescia, Viale Europa 11, 25123 Brescia, Italy e-mail: bruno.cesana@unibs.it 